\title{
10 MVA Thyristor Converters for the Active Damping of Generator Shaft Oscillations at ASDEX Upgrade
}

\author{
C.-P. Käsemann ${ }^{1}$, L. van Lieshout ${ }^{2}$, M. Huart ${ }^{1}$, M. Rott $^{1}$ and the ASDEX Upgrade Team \\ ${ }^{1}$ Max-Planck-Institut für Plasmaphysik (IPP), EURATOM Association, \\ Boltzmannstraße 2, D-85748 Garching, Germany \\ 2 Imtech Vonk BV, Modem 30, NL-7741 MJ Coevorden, The Netherlands
}

\begin{abstract}
Over the last years, ASDEX Upgrade (AUG) improved its fast plasma feedback control. This had a direct implication on the magnetic coils power supplies. During a discharge, very fast changes of the active power occur. This results in torsional oscillations in the flywheel generators shaft lines that feed the power supplies. These oscillations speed up the aging of the generator assemblies. Therefore an active control system providing electromagnetic damping for the generator shaft oscillations has been set up. To optimize this system, two new thyristor converters have been installed and commissioned.
\end{abstract}

Especially the new "design features" of the Thyristor Converter Groups 8 and 9 with generator torque reference processing and protection will be explained in the paper. Further on, the paper will present the various modes of operation, analyses the results of measurements obtained during commissioning, compares them to the calculated (design) values and reports on the performance achieved during AUG plasma experiments.

Keywords: ASDEX Upgrade; Flywheel generator; Torsional resonance; Active damping; Magnetic energy storage; Power supply; Thyristor converter

\section{Introduction}

The ASDEX Upgrade (AUG) tokamak requires electrical power up to a few hundred MW for a time period of about $10 \mathrm{~s}$. The power and energy are provided by three separate networks based on flywheel generators as shown in Fig. 1. In 1999, during a routine check performed on generator EZ3 (144 MVA / $544 \mathrm{MJ})$, it was discovered that the

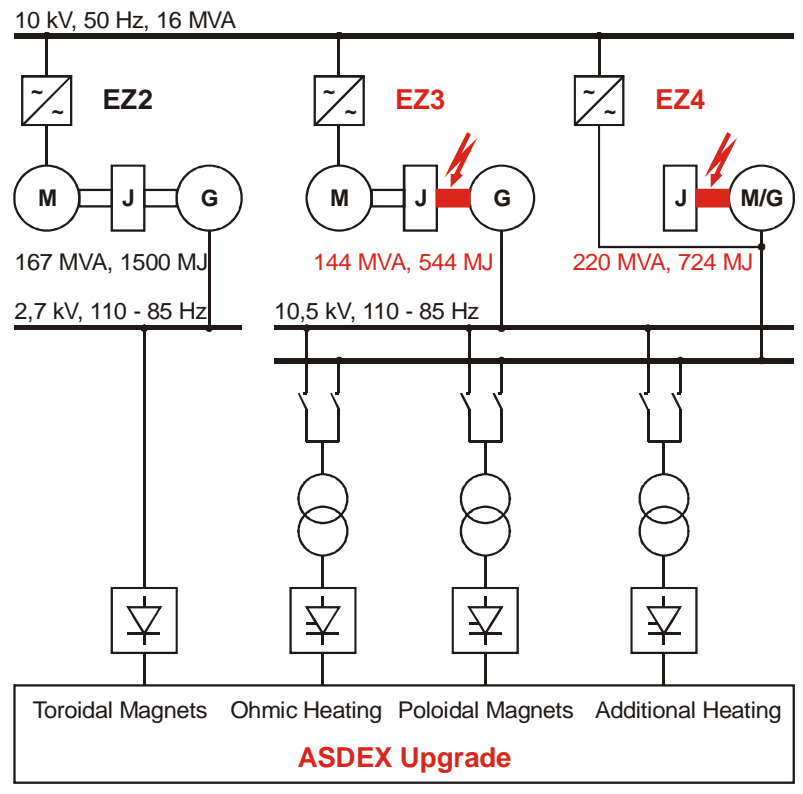

Fig. 1 AUG power supply by flywheel generators coupling bolts of the flywheel-generator shaft were deformed. In 2002, damage was also discovered during inspection of the coupling of the EZ4 (220 MVA / $724 \mathrm{MJ}$ ) shaft assembly (Fig. 2) [1]. The damages at the generators have been explained by a torsional resonance of the shaft, excited by active power transients from the converter loads. Values of $23.8 \mathrm{~Hz}$ and $26 \mathrm{~Hz}$ were calculated for the first natural frequency of the EZ3 and EZ4 shaft assembly. These

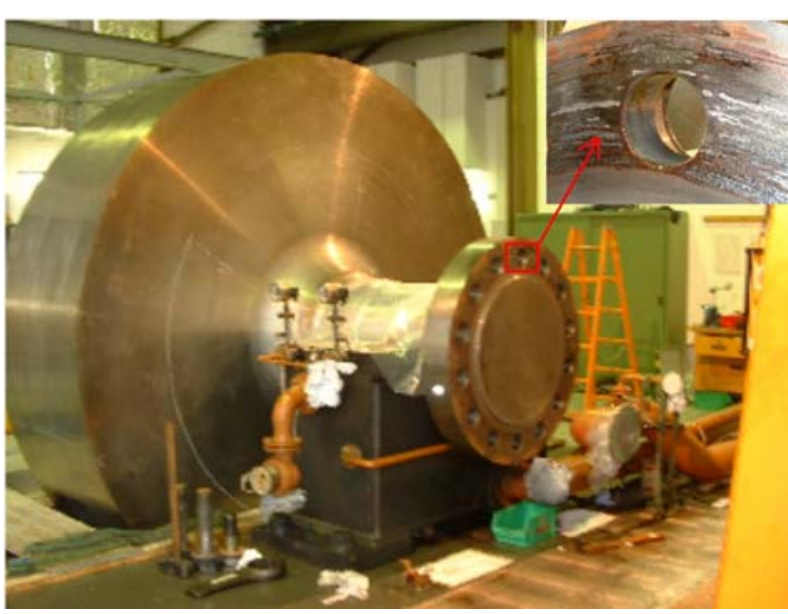

Fig. 2 Damage at coupling of EZ4 frequencies could be found in the spectrum of the AUG load curves.

\section{Application}

In 2001, contactless torque measurement systems were installed close to the coupling between the flywheel and the rotor of the EZ3 and EZ4 generators. Low trip levels of the torque signals had to be set to prevent further damages on the generator shaft system, resulting in a large number of early terminated AUG pulses.

To reduce the torsional stresses in the generator shafts, IPP decided to develop a new method of damping. The IPP damping circuits were designed to damp torsional resonances by means of active power provided by magnetic energy storage units. 
However, the design of the damping system and control scheme must not influence the control parameters of the network or tokamak load. The block diagram of the damping circuit is shown in Fig. 3. The theoretical background, simulation model and numerical investigations of the developed damping circuit are fully described in [2].

First, the damping system was based on two thyristor converter modules of the AUG power supply $\left(\mathrm{U}_{\text {dio }}=1.6 \mathrm{kV}, \mathrm{I}_{\mathrm{N}}=22.5 \mathrm{kA}\right)$. For magnetic energy storage the IPP high current converters test load $(\mathrm{L}=5 \mathrm{mH})$ and spare coils with much lower inductance $(2 \times 1 \mathrm{mH})$ were used. With this installation, the damping capability of the circuit was demonstrated in 2003 and used for damping the EZ3 and EZ4 generators during AUG operation thereafter.

The converters used were highly overrated and will be needed for future AUG long pulse experiments. The coils were not adapted best for the damping task. But the biggest disadvantage was that the control circuits of the damping were integrated in the

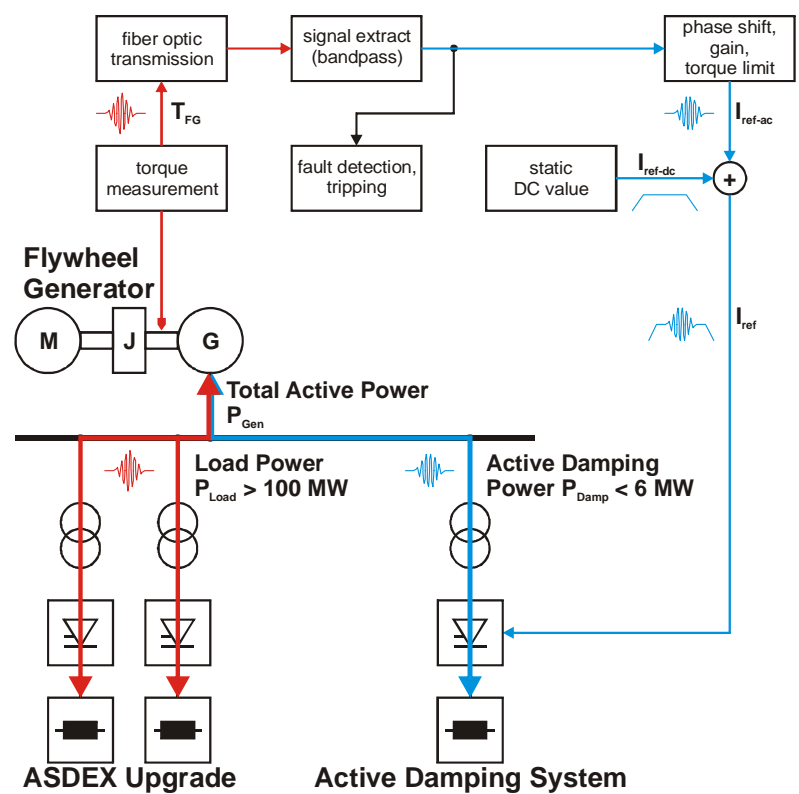

Fig. 3 Block diagram of the active damping circuit AUG control system. This meant that the damping system was not available for AUG independent tests of e.g. the additional heating systems $(\mathrm{AH})$ or other installations.

Therefore in 2004 the basic parameters for an integrated damping system with independent control circuits, thyristor converters and damping coils were identified for the generators EZ3 and EZ4.

\section{Converter Concept}

In a first step, two optimized air cored coils were purchased and commissioned in 2005. In the same year the contract for two dedicated thyristor converters including signal processing and damping control system were settled.

The converters and their control include several new features derived from the experience accumulated with the operation of the "prototype" system. They have to fulfil three main objectives: monitoring the torsional oscillations - to protect the generators against excessive torque; signal conditioning - to achieve the correct phasing of damping power; pulsed or continuous damping of the torsional oscillations - to provide maximum short time damping power or to avoid resonance stimulations at low power.

\section{a) Monitoring and Protection}

Via an optical link, the control unit is directly connected to the generator's torque sensors. To prevent excessive torque, both sensors $\mathrm{M} 1$ and $\mathrm{M} 2$ are compared with a maximum set level. To detect faulty sensors, the difference of the two signals (M1-M2) is monitored, too. As soon as the auxiliaries are switched on and the generator is connected to the busbar, the protection is working. This means that even if the active damping is disabled - the generator will be disconnected from the busbar as soon as one of the limits is exceeded.

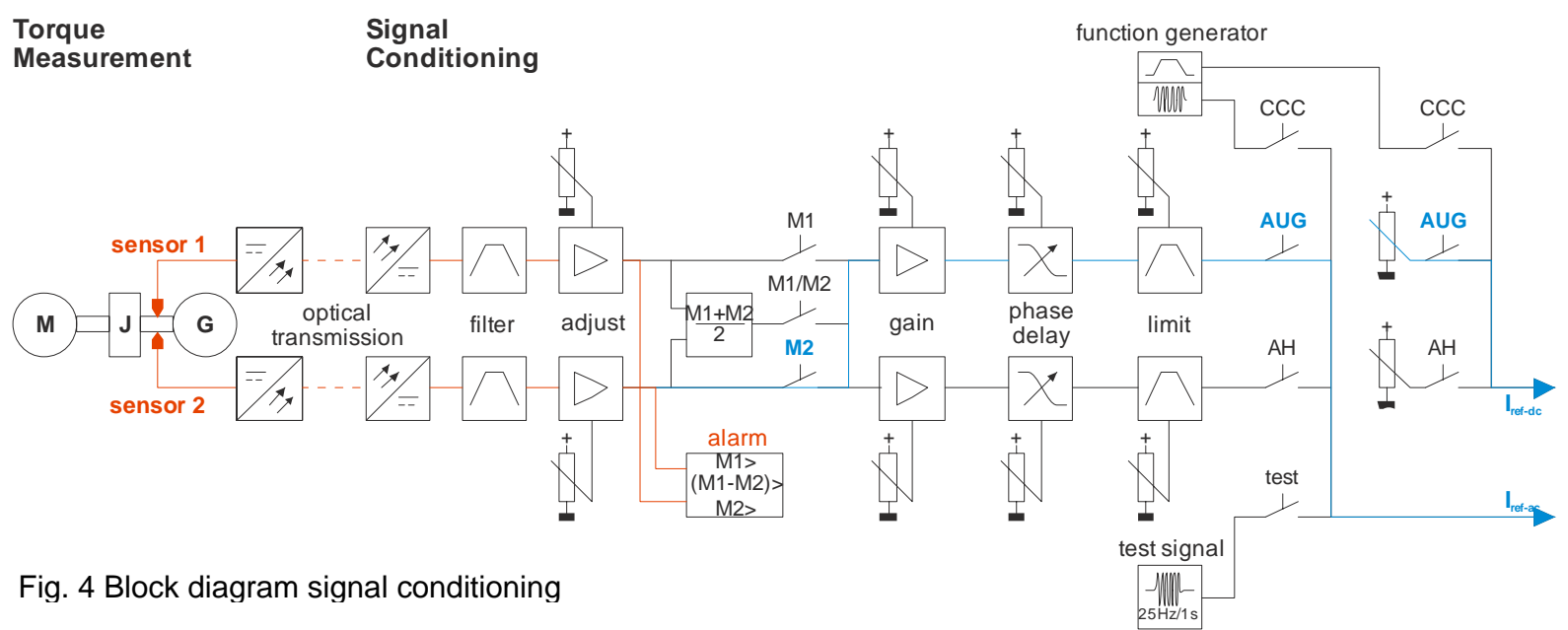




\section{b) Signal Conditioning}

The block diagram of the signal conditioning is given in Fig. 4. With the new system it is possible to connect both sensor signals M1 or M2 to the damping unit. Further on, the average of both signals (M1+M2)/2 can be used. The dc offset of the raw torque signal is removed by a first order high-pass filter having a cut-off frequency of $10 \mathrm{~Hz}$, followed by a second order low-pass Bessel filter with a cut-off frequency of $40 \mathrm{~Hz}$ to remove high frequency disturbances. The remaining ac signal is phase shifted in order to produce the reference ac signal for the thyristor converter. For optimum damping performance a total phase shift of $180^{\circ}$ is needed between the exciting torque and the damping torque (see Fig. 5). The advantage of this feedback circuit is that the current flow in the coil is controlled with a frequency corresponding to the measured natural frequency of the shaft assembly. Therefore, with proper phasing, only very little power needs to be applied in order to provide sufficient damping for the problems experienced in the past. After signal limiting and gain setting the phase-shifted reference ac signal is superimposed on a static dc value. The gain and dc value depend on the mode of operation. At AUG operation the system is set for maximum power short time damping. For standalone operation of additional heating systems $(\mathrm{AH})$, the gain and dc set level are reduced to provide continuous low power damping. The combined ac and dc signal is used as reference signal to the thyristor converter.

For testing purpose it is possible to change over from the ac torque reference signal to a fixed ac signal that is trimmed to the generator resonant frequency. With this signal a defined excitation of the generator torque for a time period of $0.5 \mathrm{~s}$ is possible. The damping following there after can be examined and compared to earlier measurements (see Fig. 6), for system calibration purposes.

Further on, a function generator can be connected to the signal conditioning board allowing for independent ac and dc reference test signals.

\section{c) Pulsed and Continuous Damping}

The reference signal coming from the signal conditioning board controls the DC current of a 6-pulse thyristor converter. The converter feeds a DC load coil used as buffer storage of magnetic energy, being loaded and unloaded in counter-phase to the torsional velocity of the shaft.

The converters are directly connected to the $10.5 \mathrm{kV}, 85-110 \mathrm{~Hz}$ networks of the generators EZ3 and EZ4 and are completely independent from other systems. The converters are designed and tested to be short- circuit proof on their DC terminals. The converter transformers have fixed voltage ratios. The noload voltage is $1.6 \mathrm{kV}$. The voltage loop bandwidth of the converters is $150 \mathrm{~Hz}$. The nominal rating of every converter is $5 \mathrm{kA}$-dc plus $1 \mathrm{kA}$-ac for pulsed damping (AUG) and $1.5 \mathrm{kA}$-dc plus $0.5 \mathrm{kA}$-ac for continuous damping (AH).

\section{Commissioning}

Prior to the installation at site, the main components were type- and routine tested in the factory. The transformer was tested into a short-circuit condition. Thyristors have been tested for their reverse recovery and turn-off characteristics. After erection, comprehensive tests have been carried out in noload, light load (10 $\Omega$ series resistor), pulsed load and short-circuit conditions.

After commissioning of the converters and their local control and feedback circuits, the various functions of the torque reference signal conditioning board have been adjusted. Finally, the installation has been successfully tested under real experimental conditions.

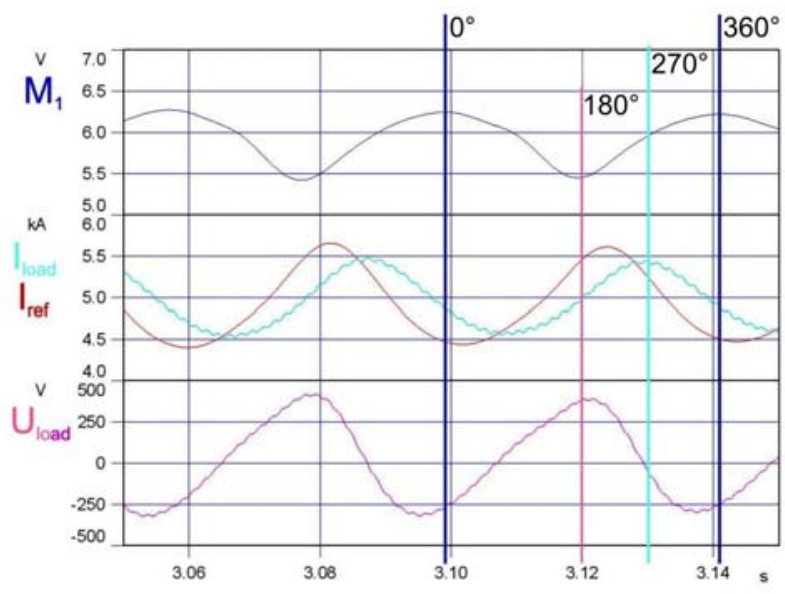

Fig. 5 Phase relationship

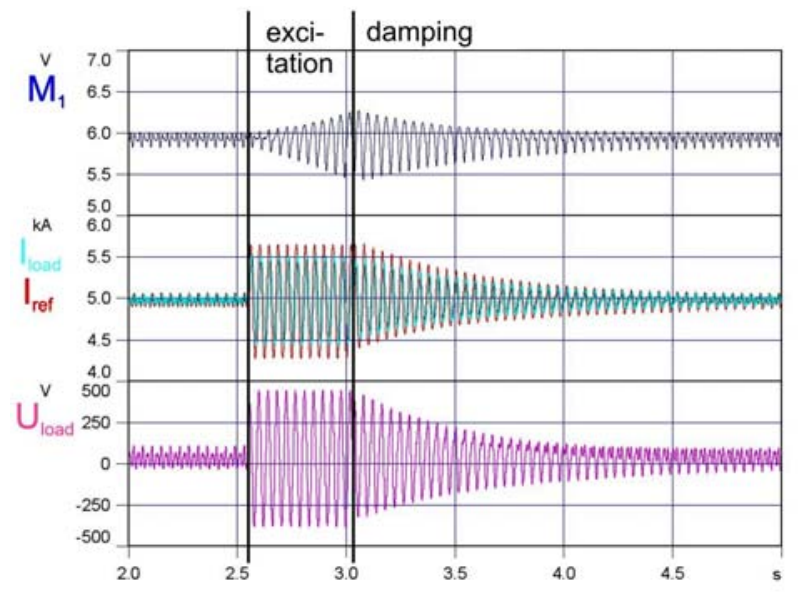

Fig. 6 Testing of excitation and damping 


\section{Operational Experience}

The commissioning of the thyristor converter Group 8 representing the active damping system of generator EZ3 has been completed in summer 2007. It was found that the system works very well under the various AUG pulse scenarios. Up to now the effectiveness and robustness of the new equipment has been proven in more than 1500 plasma discharges. An example is given in Fig. 7. This pulse has been finished early by an AUG pulse-stop (decrease of $I_{\text {gen }}$ ). At this moment the damping is switched off, too, and the torque at the generator stays at a high level for quite a long time.

Since generator EZ4 was not available, the thyristor converter Group 9 has been commissioned on EZ3, too and is ready for operation. Only the signal conditioning circuits need to be adjusted to the EZ4 generator later on.

The maximum torque during AUG operation at generator EZ3 with the temporary damping circuit (Group 6) and with the new installation (Group 8) can be found in Fig. 8. At the few trips because of excessive torque it was noticed that the rate of rise of the torque was so fast, that the protection tripped before the damping system could react. Since the torque reference signal generation proved its reliability, a time delay of the trip signal of about $100 \mathrm{~ms}$ seems to be possible in the future. This would allow for the damping converters to react on the torsional resonance without overload of the generator.

So far, continuous damping during $\mathrm{AH}$ operation has not been used. Anyhow, during commissioning, quite excessive torque excitations by the $\mathrm{AH}$ systems have been noticed (see Fig. 9). It has turned out that there were Neutral Beam conditioning pulses at a pulse rate of exactly the generator's torsional resonant frequency being $24 \mathrm{~Hz}$.

\section{Conclusion}

A novel system for damping resonances in a generator shaft line has been developed, installed, commissioned and operated since 2003. The temporary installation has been replaced by an optimized and dedicated converter and coil assembly with independent signal conditioning and protection. It is directly connected to the generator busbars and damps the first torsional mode efficiently, providing torques to the rotor-shaft system which have the same effect as increased natural damping. The active damping circuits are independently controlled by using the torsional stress measured by contactless torque sensors. The active power for damping the torsional resonances is taken from compact buffer storages of magnetic energy. They are loaded and unloaded with the natural frequency of the first torsional mode of the shaft assembly, thus providing sufficient damping power in generator shafts weighing more than $100 \mathrm{t}$.

\section{References}

[1] C. Sihler et al., Excitation of Torsional Oscillations in Generator Shaft Lines by Plasma Feedback Control, SOFT, Helsinki 2002, Fusion Eng. Des. 66-68 (2003) 1061-1064.

[2] C. Sihler et al., Damping of Torsional Resonances in Generator Shafts Using a Feedback Controlled Buffer Storage of Magnetic Energy, IPST, New Orleans 2003

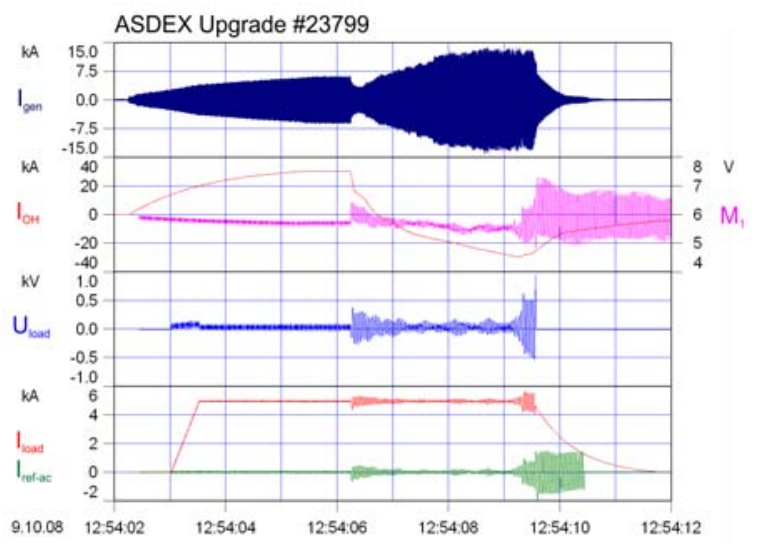

Fig. 7 Damping during AUG plasma pulse

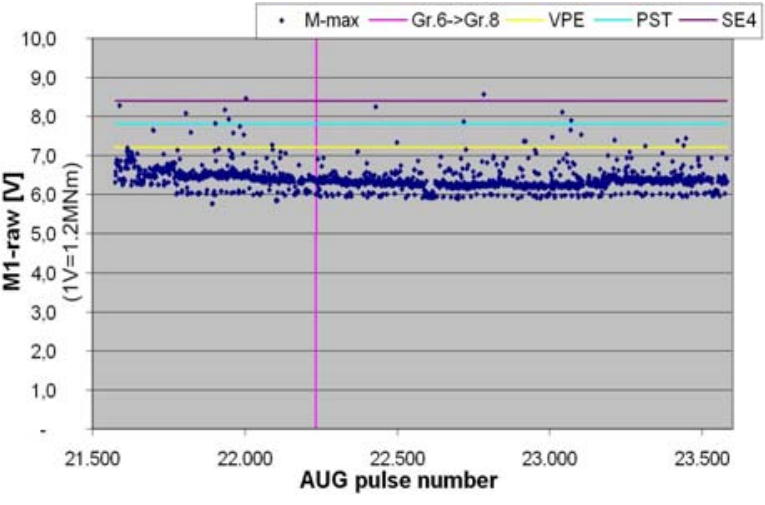

Fig. 8 Maximum torque statistics

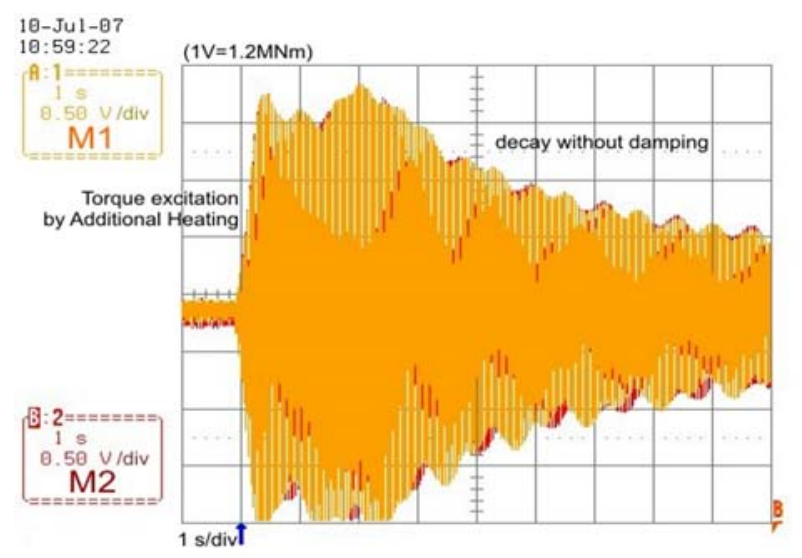

Fig. 9 Torque excitation during NBI conditioning 